\title{
Active gesture-changeable underactuated finger for humanoid robot hand based on multiple tendons
}

\author{
D. Che and W. Zhang
}

Key Laboratory for Advanced Materials Processing Technology, Ministry of Education, Department of Mechanical Engineering, Tsinghua University, Beijing 100084, China

\begin{abstract}
Received: 27 February 2010 - Revised: 25 June 2010 - Accepted: 19 August 2010 - Published: 22 December 2010
\end{abstract}
\begin{abstract}
The concept called gesture-changeable under-actuated (GCUA) function is utilized to improve the dexterities of traditional under-actuated hands and reduce the control difficulties of dexterous hands. Based on GCUA function, a novel mechanical finger by multiple tendons: GCUA-T finger, is designed. The finger uses tendon mechanisms to achieve GCUA function which includes traditional underactuated (UA) grasping motion and special pre-bending (PB, or pre-shaping) motion before UA grasping. Operation principles and force analyses of the fingers are given, and the effect of GCUA function on the movements of a hand is discussed. The finger can satisfy the requirements of grasping and operating with low dependence on control system and low cost on manufacturing expenses, which develops a new way between dexterous hand and traditional under-actuated hand.
\end{abstract}

This paper was presented at the IFToMM/ASME International Workshop on Underactuated Grasping (UG2010), 19 August 2010, Montréal, Canada.

\section{Introduction}

In recent years, breathtaking developments in robotics have been witnessed all over the world. The field of robotic hands including dexterous hands and under-actuated hands are emphasized and developed.

Over the past 30 years, researchers have made plentiful achievements on the study of dexterous hand. For instance, Stanford/JPL dexterous hand was designed and analyzed by J. Salisbury et al. (1982), which has three 3-DOF fingers actuated by 12 DC motors, each joint of this hand can be flexed and extended independently by one actuator. Gifu II hand, designed by H. Kawasaki et al. (1986), has 5 fingers whose all joints are actuated by servomotors, which can perform dexterous object manipulation like the human hand; Utah/MIT dexterous hand was designed by S. Jacobsen et al. (1992), which has four 4-DOF fingers with 32 independent tendons and 32 pneumatic cylinders (2004), the hand can be used as a high flexible tool for the study of machine dexterity. Dexterous hand can do almost all the movements and gestures of human hand. In fact, almost each DOF of a dexterous hand need an actuator to drive, which makes the hand high depend on control and high cost on manufacturing and using expenses.

On the contrary, under-actuated hands use fewer motors to drive more DOFs, and the under-actuated hands have a very amazing feature: self-adaptation in grasping, which let the hands easy to control. Many studies have done in the field of under-actuated hands: L. Birglen et al. (Birglen, 2003, 2004 and 2005), designed many kinds of under-actuated grippers and gave force analyses on them. A. Dollar et al. (Dollar, 2006 and 2007), gave a SDM robust robotic grasper which uses a single actuator to drive 8 DOFs; L. Tan et al. (2009), designed a multi-fingered hand using hydraulic actuation with fluidic actuators, the hand has 14 DOFs which can bend when hydraulic pressure is applied by a water pump.

This paper introduced the concept called gesturechangeable under-actuated (GCUA) function. Based on GCUA function, a new robot finger was designed for humanoid robot hands based on multiple tendons. 


\section{GCUA function}

Traditional under-actuated (UA) function can drive many DOFs of a robotic hand with motors whose number is less than that of the hand's DOFs. There are two main kinds of under-actuated hands with traditional UA function:

1. When a hand grasp an object, it cannot change its initial gesture which is fixed (almost keep straight) until its phalange touches some objects. An example of this kind of under-actuated hand is TH-3 hand which designed by Tsinghua University (Zhang, 2009). TH-3 hand is based on gear-rack transmission, which cannot bend its phalanges without resistances of objects, this makes it feel hard to grasp gliding objects stably.

2. When a hand grasp objects, all or part of its joints must bend in a fixed order which cannot be changed when the mechanism has been designed. For instance, a multi-function mechanical hand with shape adaptive which was designed by G. Guo, X. Qian, and A. Gruver (1993), is based on link and gear transmission. The hand can grasp different objects with shape adaptive function. However, with gear transmission, the rotating angles of middle joint and distal joint are proportional, which limits its dexterity.

Traditional UA function can make robotic hand low depend on sensing and control, however, it cannot ensure the hands enough flexibility to grasp or operate. A concept called gesture-changeable under-actuated (GCUA) function was proposed (Zhang, 2009) to improve the dexterities of traditional under-actuated hands and reduce the control difficulties of dexterous hands. The GCUA function includes two main motions: under-actuated (UA) motion and pre-bending (PB, or pre-shaping) motion, which are shown in Fig. 1.

UA motion preserves advantages of traditional underactuated hand, which makes the hand low depend on senor and control system. With UA motion, the hand can grasp different objects self-adaptively.

The hand can change its initial gesture with PB motion, which can achieve different grasping postures. After it has got a satisfied posture, the hand can grasp different objects with UA motion. PB motion is extremely important when high grasping dexterity and stability are required.

\section{Design of GCUA-T fingers by tendons}

3-joint GCUA-T finger uses tendon mechanism to achieve GCUA function, which is shown in Fig. 2.

Proximal joint is fixed in base, which is between the base and 1st middle phalange; middle joint is fixed in 1st middle phalange, which is between 1st and 2nd middle phalanges; distal joint is fixed in 2nd middle phalange, which is between 2nd middle phalange and distal phalange; UA transmission and PB transmission are both fixed in base; 1st return spring

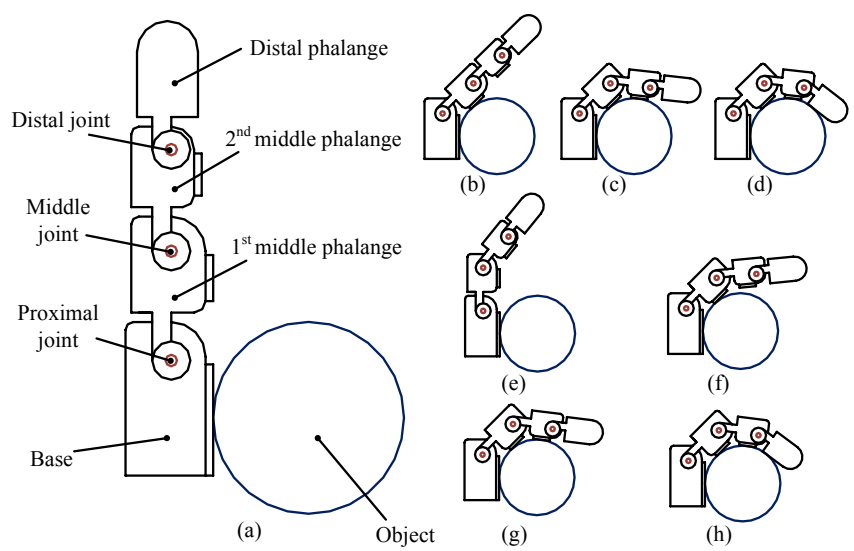

Figure 1. UA motion and PB motion of 3-joint GCUA finger. (a, b, c, d) UA motion; (a, e) PB motion; (f, g, h) UA motion with the changed initial gesture; (a) Initial gesture (keep straight); (b) Proximal joint rotates until 1st middle phalange touches the object; (c) Middle joint rotates until 2nd middle phalange touches the object; (d) Distal joint rotates until distal phalange touches the object; (e) Middle joint rotates to change the finger's initial gesture.

combines 2nd middle phalange and distal phalange with its two ends, similarly 2 nd return spring combines 1 st middle phalange and 2nd middle phalange with its two ends.

Assembly methods of UA tendon, RS tendon, and PB tendon are shown in Fig. 3.

UA tendon combines UA transmission and distal phalange with its two ends, which wraps around distal joint, middle joint and proximal joint; RS tendon uses its two ends to combine 1st middle phalange and UA transmission, which wraps around proximal joint and cross through the base; $\mathrm{PB}$ tendon combines 2nd middle phalange and PB transmission with its two ends, which wraps around middle joint and proximal joint; The wrapping directions of UA tendon and $\mathrm{PB}$ tendon are the same, which are different from that of RS tendon, so that UA tendon and PB tendon will drive the finger to be flexed, on the contrary, RS tendon will drive the finger to be extended. PB tendon can also be assembled in another method, which is shown in Fig. 3d.

\section{The work process of GCUA-T finger}

With UA tendon and RS tendon, UA motion will proceed in this way: 1st motor rotates forward, UA transmission pulls UA tendon and RS tendon to move down. Since the wrapping directions of these two tendons are different, UA tendon will tension, simultaneously RS tendon will relax; In this case, UA tendon drives distal phalange to rotate around distal joint. With the constraint of 1 st return spring and 2nd return spring, 1st middle phalange, 2nd middle phalange and distal phalange rotate around proximal joint together as a rigid body. When 1st middle phalange is blocked by objects, 2nd middle phalange and distal phalange will rotate around middle 


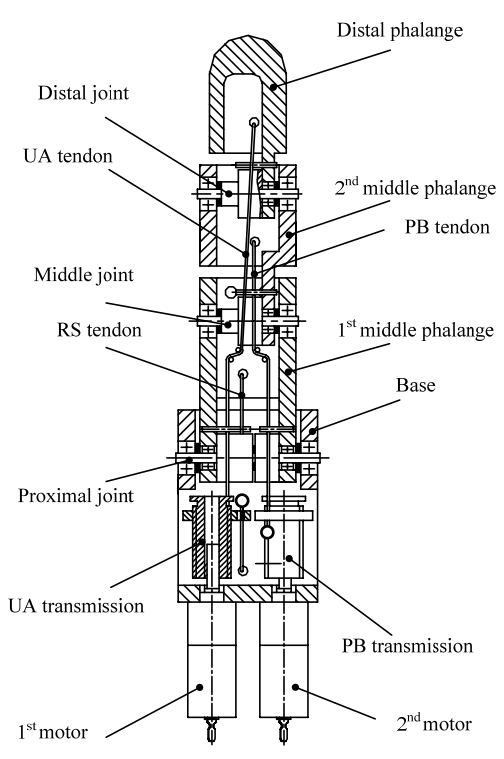

(a)

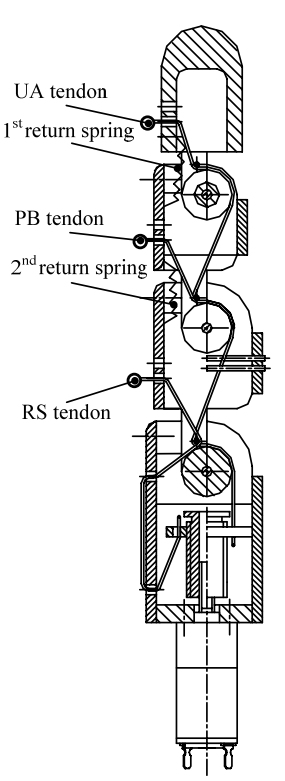

(b)

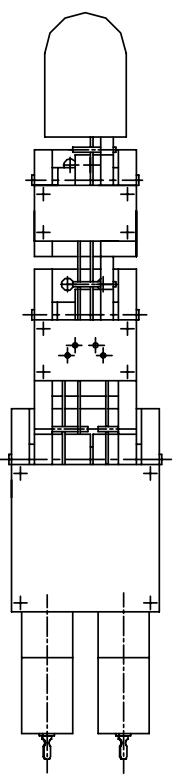

(c)

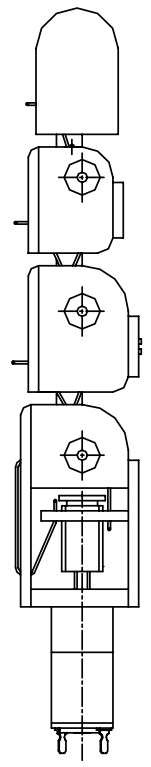

(d)

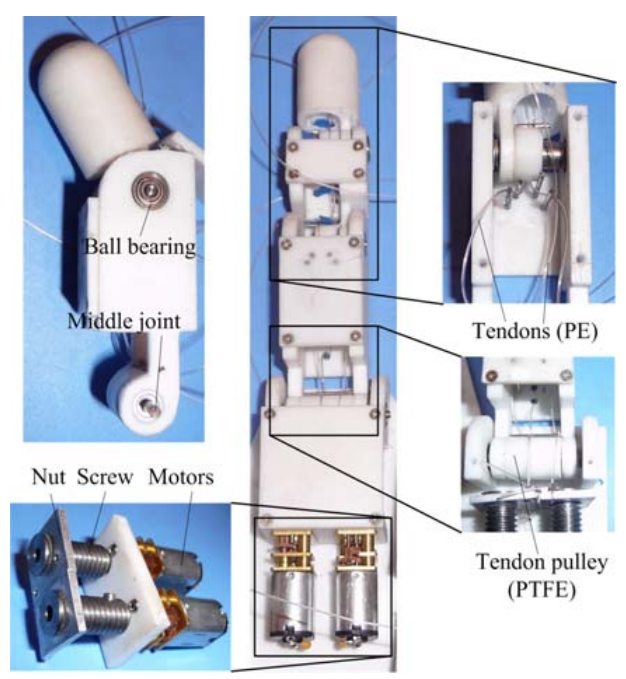

(e)

Figure 2. Structure of 3-joint GCUA-T finger. (a) Front cutaway view; (b) Side cutaway view; (c) Front view; (d) Side view; (e) 3-joint GCUA-T finger.

joint together, simultaneously 2 nd return spring will extend; Similarly, when 2nd middle phalange is blocked by objects, distal phalange will rotate around distal joint, and 1st return spring will extend. In this way, the finger can grasp objects self-adaptively.

When 1st motor rotates back, UA transmission will pull these two tendons up, so that UA tendon will relax, and RS tendon will tension. RS tendon will drive the finger to extend and leave the object. When UA transmission goes back to the initial status, the finger will be back to the initial gesture too.
If $\mathrm{PB}$ tendon is assembled in the method which is shown in Fig. 3b, PB motion will proceed like this: When 2nd motor rotates forward, $\mathrm{PB}$ transmission pulls $\mathrm{PB}$ tendon to change the finger's initial gesture, since RS tendon prevents 1st middle phalange from bending and 1st return spring makes the top two phalanges become a rigid body, 2 nd middle phalange and distal phalange will rotate around middle joint together, so that the finger can change its initial gesture and then grasp things with UA motion. In this process of PB motion, if either of middle phalange and distal phalange is blocked by 


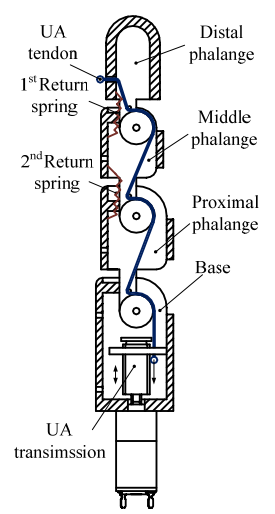

(a)

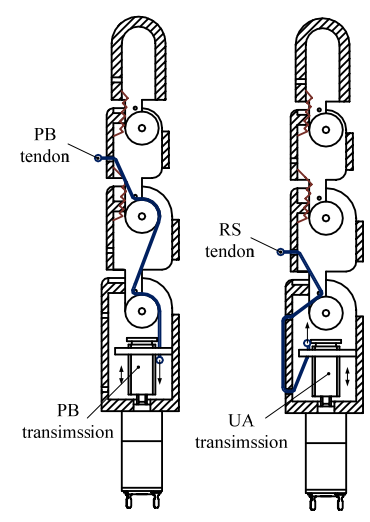

(b)

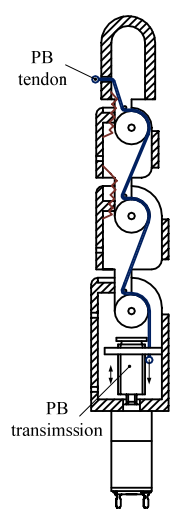

(d)
Figure 3. Assembly method of finger's tendons. (a) UA tendon; (b) PB tendon; (c) RS tendon; (d) another assembly method of PB tendon.

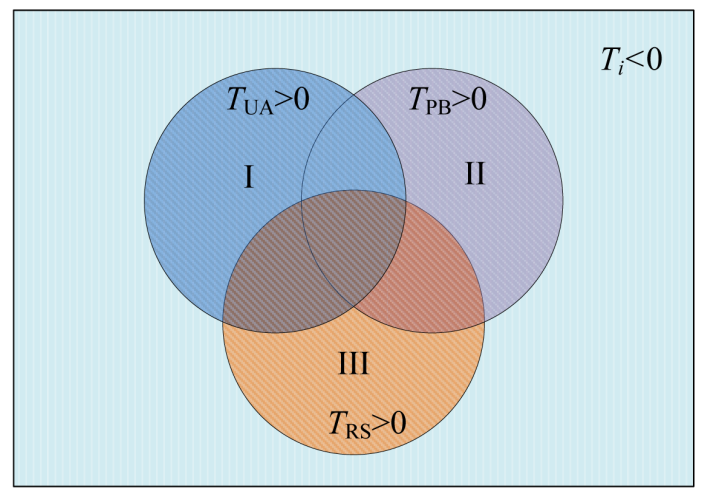

Figure 4. Operation areas of GCUA-T 3-joint finger.

objects, the finger will stop bending. However, when PB tendon is assembled in another method which is shown in Fig. 3b, PB motion will change. In this case, if 2 nd middle phalange is blocked by objects, distal phalange can keep rotating around distal joint until it touches objects.

When 2nd motor rotates back, PB transmission will pull PB tendon up, and PB tendon will relax, after that, return springs will drive the top two phalanges to go back to their initial status.

Indeed, tendons can only restrict the movement of the finger in a single-direction, so that if one tendon relaxes, it will not have effect on other tendons, in this way, UA motion and $\mathrm{PB}$ motion can proceed independently. Assume $T_{\mathrm{UA}}, T_{\mathrm{PB}}$, and $T_{\mathrm{RS}}$ as the driving torques of UA tendon, $\mathrm{PB}$ tendon and RS tendon with regard to proximal joint, and suppose that when $T_{i}>0$, the tendon will tension, on the contrary, when $T_{i}<0$, the tendon will relax, the operation areas can be given, which is shown in Fig. 4.

In area I, since $T_{\mathrm{UA}}>0, T_{\mathrm{RS}}<0$, and $T_{\mathrm{PB}}<0$, both RS tendon and $\mathrm{PB}$ tendon relax, UA tendon tensions, so that UA

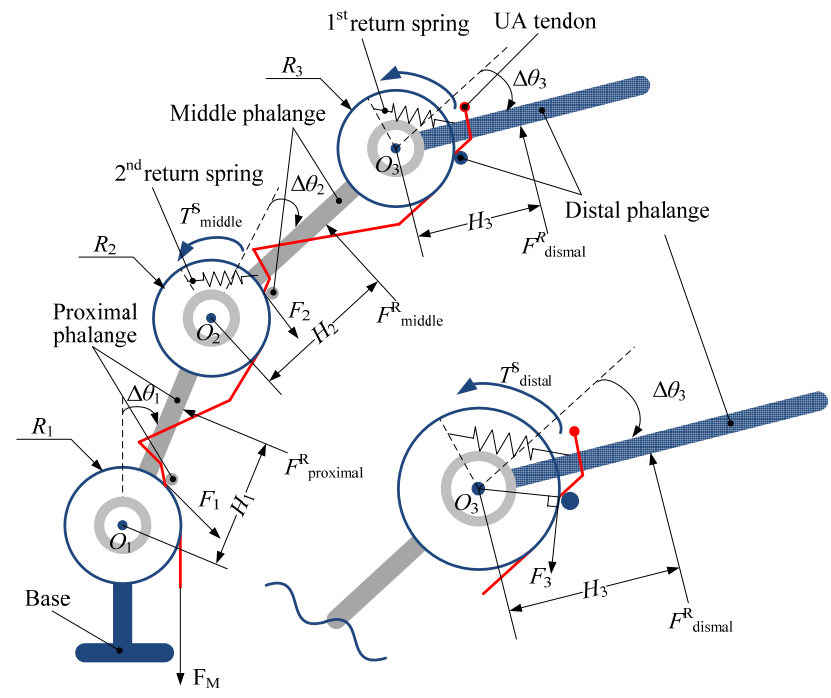

(a)

(b)

Figure 5. Force analysis of GCUA-T finger when UA motion proceeds. (a) Force analysis on the whole finger; (b) Force analysis on distal phalange.

motion proceeds and the finger is flexed; In area II, since $T_{\mathrm{UA}}<0, T_{\mathrm{RS}}>0$, and $T_{\mathrm{PB}}>0$, both $\mathrm{PB}$ tendon and RS tendon tension, UA tendon relaxes, so that $\mathrm{PB}$ motion proceeds and the finger changes its initial gesture; In area III, since $T_{\mathrm{UA}}<0, T_{\mathrm{RS}}>0$, and $T_{\mathrm{PB}}<0$, both UA tendon and PB tendon relax, RS tendon tensions, so that UA motion proceeds and the finger is extended.

\section{Force analysis of GCUA-T finger}

Force analysis on GCUA-T finger is given when UA motion proceeds, which is shown in Fig. 5. When 1st motor rotates forward, UA transmission pulls UA tendon to bend the finger's phalanges. Firstly, only consider force analysis on distal phalange. When distal phalange touches objects, the objects will stop distal phalange from rotating forward with $F_{\text {distal }}^{\mathrm{R}}$. Let the torque of $F_{\text {distal }}^{\mathrm{R}}$ with regard to distal joint $\mathrm{O}_{3}$ be $T_{\text {distal }}^{\mathrm{R}}$, which can be expressed as:

$T_{\text {distal }}^{\mathrm{R}}=F_{\text {distal }}^{\mathrm{R}} H_{3}$

where $H_{3}$ is the force arm of $F_{\text {distal }}^{\mathrm{R}}$ with regard to distal joint $\mathrm{O}_{3}$.

Considering the physical property of UA tendon, the tendon's tension has the same magnitude in any point of the whole tendon, i.e.

$F_{3}=F_{\mathrm{M}}$

UA tendon drives distal phalange to bend with $F_{3}$ which is resulting force of UA tendon's tension $F_{\mathrm{M}}$. In this case, the 
driving torque $T_{\text {distal }}^{\mathrm{M}}$ caused by UA tendon with regard to distal joint $O_{3}$, which can be given by

$T_{\text {distal }}^{\mathrm{M}}=F_{\mathrm{M}} R_{3}$

Let the angle between distal phalange and 2nd middle phalange be $\Delta \theta_{3}$, the torque $T_{\text {distal }}^{\mathrm{S}}$ caused by 1 st return spring with regard to distal joint is obtained as follows,

$T_{\text {distal }}^{\mathrm{S}}=-K_{1} \Delta \theta_{3}$

where $K_{1}$ is the stiffness of 1 st return spring.

Force analysis on other phalange is similar with that on distal phalange, when 1st middle phalange and 2nd middle phalange are blocked by objects, the objects will prevent them from bending with $F_{\text {proximal }}^{\mathrm{R}}$ and $F_{\text {middle }}^{\mathrm{R}}$, the force arm of $F_{\text {proximal }}^{\mathrm{R}}$ with regards to proximal joint $O_{1}$ is $H_{1}$, similarly the force arm of $F_{\text {middle }}^{\mathrm{R}}$ with regards to middle joint $\mathrm{O}_{2}$ is $\mathrm{H}_{2}$, the following equation can be obtained,

$\boldsymbol{T}^{\mathrm{R}}=J_{P} F^{\mathrm{R}}$

where $\boldsymbol{T}^{\mathrm{R}}$ is the external resisting torque vector, $\boldsymbol{F}^{\mathrm{R}}$ is the external resisting force vector, i.e.

$\boldsymbol{F}^{\mathrm{R}}=\left[\begin{array}{c}-F_{\text {proximal }}^{\mathrm{R}} \\ -F_{\text {middle }}^{\mathrm{R}} \\ -F_{\text {dismal }}^{\mathrm{R}}\end{array}\right]$

$J_{P}=\left[\begin{array}{lll}H_{1} & 0 & 0 \\ 0 & H_{2} & 0 \\ 0 & 0 & H_{3}\end{array}\right]$

When UA transmission keeps still, if external force drive 1st middle phalange to bend, there will be a resisting torque $T_{\mathrm{RS}}^{\mathrm{R}}$ caused by RS tendon. Let the angle between the base and 1st middle phalange be $\Delta \theta_{1}$, the angle between 1st middle phalange and 2nd middle phalange be $\Delta \theta_{2}$, the following equation can be obtained,

$\boldsymbol{T}^{\mathrm{S}}=\left[\begin{array}{l}-T_{\mathrm{RS}}^{\mathrm{R}} \\ -K_{2} \Delta \theta_{2} \\ -K_{1} \Delta \theta_{3}\end{array}\right]$

where $\boldsymbol{T}^{\mathrm{S}}$ is the internal resisting torque vector, $K_{2}$ is the stiffness of 2 nd return spring.

Considering the principle of tendon mechanism, the relationship among $\Delta \theta_{1}, \Delta \theta_{2}$, and $\Delta \theta_{3}$ can be given,

$\Delta \theta_{1} R_{1}+\Delta \theta_{2} R_{2}+\Delta \theta_{3} R_{3}=\Delta L$

where $R_{1}, R_{2}$ are the radii of proximal joint and middle joint respectively, and $\Delta L$ is the displacement of UA tendon's distal end from initial status to current status.

According to the driving torque caused by UA tendon, the following equation can be given,

$\boldsymbol{T}^{\mathrm{M}}=F_{\mathrm{M}} V$ where $\boldsymbol{T}^{\mathrm{M}}$ is the driving torque vector caused by UA tendon.

$V=\left[\begin{array}{l}R_{1} \\ R_{2} \\ R_{3}\end{array}\right]$

Considering force balance and torque balance of the whole finger, if the finger keeps still, the following equation must be satisfied,

$T^{\mathrm{M}}+T^{\mathrm{R}}+T^{\mathrm{S}}=0$

Let $\mathbf{S}=T^{\mathrm{M}}+T^{\mathrm{R}}+T^{\mathrm{S}}$, the grasping stability can be determined by matrix $\mathbf{S}$. When the elements of matrix $\mathbf{S}$ are all more than zero, the finger can grasp objects stably with UA motion. If some elements of matrix $\mathbf{S}$ are less than zero, the finger cannot grasp objects stably only with UA motion. In this case, PB motion will be proceeded to improve the grasping stability of the finger.

\section{Conclusions}

In order to make the under-actuated hand more dexterous and human-like, GCUA function is utilized into a new finger through tendon mechanisms, which consists of two motions: UA motion and PB motion. With GCUA function, the finger can change its initial gesture with PB motion and grasp objects self-adaptively with UA motion, at the same time, the cooperation of UA motion and PB motion enables the finger to do some simple gestures.

With force analysis on UA tendon of 3-joint finger, some stability requirements are given.

GCUA-T fingers based on the GCUA function develop a new way between dexterous hand and traditional underactuated hand.

Acknowledgements. This paper was funded by the National Natural Science Foundation of China (No. 50905093), Hi-Tech R\&D Program (2007AA04Z258) and the foundation of National Innovation Experiment Program for University Students (No. 091000311).

Edited by: J. L. Herder

Reviewed by: two anonymous referees

\section{References}

Birglen, L. and Gosselin, C. M.: On the Force Capability of Underactuated Fingers, Proceedings of the 2003 IEEE International Conference on Robotics and Automation, Taipei, Taiwan, September, 1139-1145, 2003

Birglen, L. and Gosselin, C. M.: Kinetostatic Analysis of Underactuated Fingers, IEEE T. Robotic. Autom., 20(2), 211-221, 2004.

Birglen, L. and Gosselin, C. M.: Fuzzy Enhanced Control of an Underactuated Finger Using Tactile and Position Sensors, Proceedings of the 2005 IEEE International Conference on Robotics and Automation, Barcelona, Spain, April, 2320-2325, 2005. 
Dollar, A. M. and Howe, R. D.: Designing Robust Robotic Graspers for Unstructured Environments, Proceedings of the Robotics: Science and Systems Conference, Workshop on Manipulation for Human Environments, Philadelphia, PA, August 2006.

Dollar, A. M. and Howe, R. D.: The SDM Hand as a Prosthetic Terminal Device: a Feasibility Study, Proceedings of the 2007 IEEE 10th International Conference on Rehabilitation Robotics, Noordwijk, The Netherlands, June, 978-983, 2007.

Guo, G., Gruver, W. A., and Qian, X.: A New Design for a Dexterous Robotic Hand Mechanism, IEEE Contr. Syst. Mag., 12(4), 35-38, 1992.

Guo, G., Qian, X., and Gruver, A.: Multi-function Mechanical Hand with Shape Adaptation, US Patent No. 5378033, 1993.

Jacobsen, S. C., Iversen, E. K., Knutti, D. F., Johnson, R. T., and Biggers, K. B.: Design of the UTAH/M.I.T. Dextrous Hand, Proceedings of the IEEE International Conference on Robotics and Automation, San Francisco, USA, April, 1520-1532, 1986.

Kawasaki, H., Komatsu, T., Uchiyama, K., and Kurimoto, T.: Dexterous Anthropomorphic Robot Hand with Distributed Tactile Sensor: Gifu Hand II, Proceedings of the IEEE International Conference on Systems, Man, and Cybernetics, Tokyo, Japan, October, 782-787, 1999.
Salisbury, J. K. and Craig, J. J.: Articulated Hands: Force Control and Kinematic Issues, The International Journal of Robotics Research, 1(4), 4-17, 1982.

Tan, L., Xie, S., Lin, I., and Lin, T.: Development of a Multifingered Robotic Hand, Proceedings of the 2009 IEEE International Conference on Information and Automation, Zhuhai/Macau, China, June, 1541-1545, 2009.

Zhang, W., Che, D., Chen, Q., and Du, D.: A Dexterous and Selfadaptive Humanoid Robot Hand: Gesture-changeable Underactuated Hand. The Second Inter Conf on Intelligent Robotics and Applications(ICIRA), 15-19 Dec 2009, Singapore, 515525, 2009.

Zhang, W., Che, D., Liu, H., Ma, X., Chen, Q., Du, D., and Sun, Z.: Super Under-Actuated Multi-Fingered Mechanical Hand with Modular Self-Adaptive Gear-Rack Mechanism, Ind. Robot, 36(3), 255-262, 2009. 